\title{
Electrical Strain Gauges, Piezoelectric Sensors or Fiber Bragg Sensors for Force Measurement: Prospects and Potentials
}

\author{
Thomas Kleckers \\ Hottinger Baldwin Messtechnik GmbH, Darmstadt Germany
}

\begin{abstract}
Two different force measurement technologies are mainly used today: Piezoelectric sensors and strain gauge based sensors. The first sensors using fiber Bragg grating technology are available now. Fiber Bragg based sensors have become increasingly important over the past years in the field of experimental stress analysis. In addition to the advantage that these sensors cannot be influenced by electromagnetic fields another major benefit is their impressive fatigue life at highest strain levels.
\end{abstract}

Key words: Force measurement, strain gauges, piezoelectric sensors, fiber Bragg technology

\section{Principles of different force sensors}

Piezoelectric transducers use the piezoelectric effect. Some known crystals produce a charge under mechanical stress. If a force is applied to such a kind of crystal the electric charge to be measured is a function of the force and the kind of crystal that is used. We can apply the fundamental equation of piezoelectric measurement:

$$
Q=q \cdot F
$$

Meanings are as follows:

$$
\begin{array}{ll}
\text { Q: } & \text { Charge to be measured } \\
\text { q: } & \text { Piezoelectric constant } \\
\text { F: } & \text { Force applied }
\end{array}
$$

Figure one shows the fundamental design of such a sensor. It features two shims made from crystal with an electrode in-between that picks up the charge.

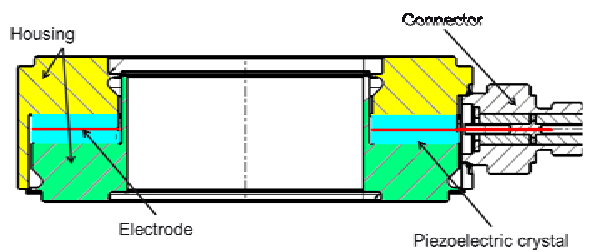

Fig.1: A piezoelectric force sensor. These sensors need to be pre-stressed for operation

The principle of strain gauge based transducers is well known and has been the leading measurement principle over the past years. A strain gauge based force transducer features a spring body to which the force is applied. The occurring mechanical stresses are converted into strain which is picked up by strain gauges. The fundamental equation of strain gauge technology shows a linear relationship between strain and change in resistance which is

$$
\frac{\Delta R}{R}=k \cdot \varepsilon
$$

Meanings are as follows:
$\Delta R:$
Change in resistance
$\mathrm{R}:$
Resistance of the strain gauge 
k: gauge

$\mathcal{E}$ :

Strain

$$
\frac{\Delta \lambda}{\lambda_{B}}=k \cdot \varepsilon
$$

Strain gauges are usually connected to a Wheatstone bridge featuring four strain gauges as a minimum. If the bridge is supplied with a voltage, the output is related to the strain occurring under the strain gauges and can be calculated as follows:

$$
\frac{U}{U_{0}}=\frac{k}{4} \cdot\left(\varepsilon_{1}-\varepsilon_{2}+\varepsilon_{3}-\varepsilon_{4}\right)
$$

\section{U: $\quad$ Supply voltage \\ $\mathrm{U}_{0}$ : $\quad$ Output voltage}

Optical force sensors work in a very similar way to strain gauge based sensors. The difference is that they use fiber Bragg gratings as sensing elements instead of electrical strain gauges.

These sensor elements feature many reflection points at regular spacing. A small portion of the light is reflected at every point. The reflected light interferes (constructive interference) and generates a reflection peak. This peak shows a characteristic wavelength that is dependent on the spacing of the reflection points.

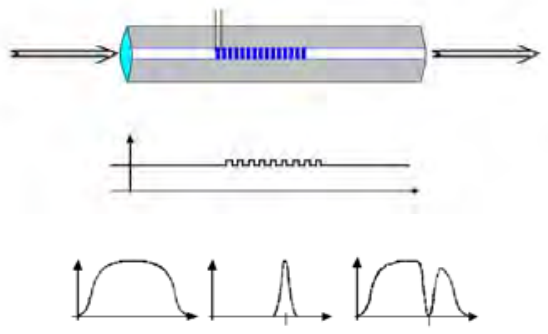

Fig.2: Fiber Bragg grating. The diagram shows the spectrum of the light that is sent into the grid (a), the reflected light (b) and the spectrum of the light in transmission

If strain is applied to a Bragg grating the spacing of the points of reflection will change. This leads to a change in wavelength of the reflected light that can be easily detected. Similar to the fundamental equation of strain gauge technology we can describe the relationship between a change in wavelength and a strain as:
Meanings are as follows:
$\Delta \lambda \quad$ Change in wavelength
$\lambda$ : $\quad$ Base wavelength
K: $\quad$ Gauge factor
$\mathcal{E}: \quad$ Strain

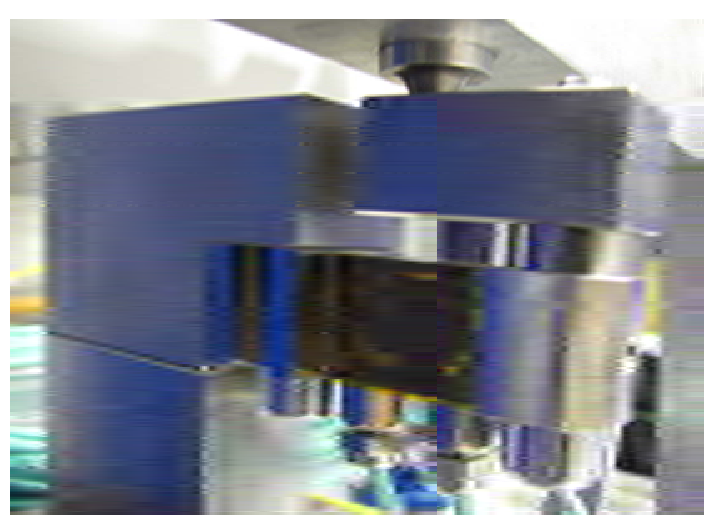

Fig.3: Optical force transducer

\section{Comparison of the characteristics of different sensors}

\section{Temperature dependency of the zero point}

Every electrical strain gauge shows a change in resistance when the temperature changes, which is based on two physical reasons:

- The material of the object - here the force transducer's spring body - will expand with higher temperatures. This is measured as a strain

- The resistance of the measuring grid material shows a change in resistance with the temperature

Modern strain gauge technology allows setting up the change in resistance in a way so that the thermal expansion coefficient can be compensated and only a very little effect can be obtained. This technology is not available with piezoelectric transducers or optical systems. 
This means that the thermal output of a strain gauge based sensor is lower than with sensors based on other principles. Using the Wheatstone bridge, this error can further be reduced dramatically as all strain gauges show the same behavior, however two of them negatively affect the output signal while the other two strain gauges have a negative influence on the output. All changes in resistance showing the same amount and the same direction of change will be compensated like the thermal output.

It is possible to use two or four optical strain gauges for one sensor using the same principle as the Wheatstone bridge. To do so, the strain signals have to be calculated in a computer using equation (3). There is the need to use positive and negative strain to have the possibility for compensation.

Because of the high sensitivity to temperature effects, fiber Bragg based sensors are very sensitive to temperature variations between the grids. Fast temperature variations and gradients strongly affect the zero point.

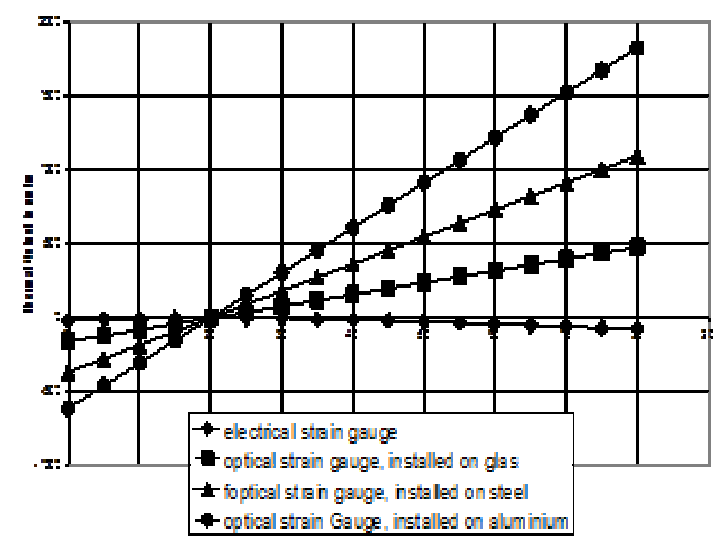

Fig.4.: Comparison of the temperature output signals of optical and electrical strain gauges.

\section{Drift}

The drift is very small with optical force transducers. As this is a frequency related measurement, drift is physically impossible. Furthermore, the sensor element is made from glass which is chemically stable.

Strain gauges have achieved quite good drift behavior over the past years as many improvements have taken place in the production process. Particularly the oxidation of measurement grids in very repeatable processes has lead to the technical standard of today's strain gauges. Monitoring over years with strain gauge based transducers is possible.
The piezoelectric sensors show a high drift. This is caused by the fact that force is turned into charge. A drift-free piezoelectric measurement chain would result in infinitely high insulation resistances that do not exist. Therefore, those sensors are limited to measurements that are not zero related or to measurement of very high forces. Those forces lead to high charges and the change in signal caused by drift becomes small relative to those signals.

\section{Creep}

A transducer's creep behavior depends on the spring material's creep behavior, which is always positive, and the creep of the sensing element, which could be an electrical strain gauge or a fiber Bragg grating.

Electrical strain gauges have a compensating effect, because their absolute value shows a negative creep that is dependent on the strain gauge's end tab length. This may be explained by the fact that force is introduced into the measuring grid through the end tabs. Here, the plastic strain gauge carrier is subjected to high shear stress, because the measuring grid on top is a spring. Due to viscoelastic processes the plastic material yields in the direction of load - the strain gauge creeps.

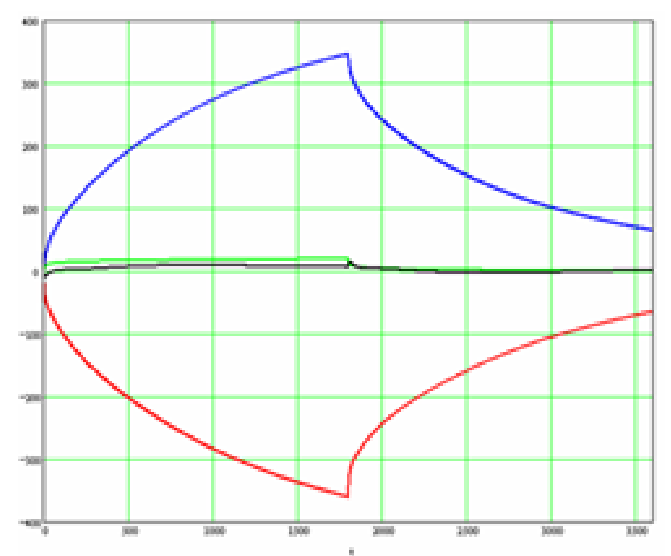

Fig. 5. Creep behavior of an electrical strain gauge: upper curve (positive creep) is the electrical strain gauge, lower curve (negative creep) is the transducer body. Total creep is zero (curve in the middle).

If very long end tabs are used, creep occurs only around the end tabs, i.e. the measuring grid links' dimensions remain unchanged and the electrical resistance and thus the measuring bridge output signal remains constant. With 
short end tabs, however, this process affects the length of the measuring grid links - the creep of the strain gauge increases. Thus, a strain gauge's creep behavior can be adapted to the creep behavior of the spring material.

Any creep compensation technology as it is known for electrical strain gauges is not available for any other force measurement technology.

Theoretically it is possible to manipulate the strain transfer from the spring body to the optical fiber to achieve creep adjustment, e.g., by changing the transfer materials. This is an objective for future development.

\section{Temperature coefficient of sensitivity}

The sensitivity of strain gauge based transducers and optical transducers changes with temperature as Young's Modulus does. This means that a higher strain level occurs at a certain load under higher temperatures: The sensors show an higher output signal.

Only strain gauge based transducers can be adjusted using temperature sensitive resistances in the wires for voltage supply of the Wheatstone bridge. If the temperature increases the resistors in the voltage supply line will have a higher resistance. This leads to a lower voltage in the Wheatstone bridge, which results in a lower output signal. The temperature influence on sensitivity is compensated.

Piezoelectric transducers show only a very low dependence on temperature and taking into account the overall accuracy of those sensors, compensation is not necessary.

Optical sensors show a very high dependence of their sensitivity on temperature but compensation can only be achieved by measurement of the temperature and calculation.

\section{Stiffness}

Every force sensor shows deflection under load. Since stiffness is defined as capacity divided by deflection, low capacity force transducers based on electrical or optical strain gauges show a very small stiffness.

Piezoelectric transducers are very stiff as crystal is a very hard material. Furthermore, those sensors always show the same sensitivity, no matter which capacity is used.

As stiffness is a very important characteristic for dynamic applications it is obvious that piezoelectric sensors offer a great advantage for dynamic measurement of low forces: The extremely stiff sensors can be used also for forces in the range of a few Newtons as their measurement range covers a very wide range. Measurement of low forces with a high capacity sensor is not a problem.

\section{Electromagnetic fields}

Electromagnetic fields of course cannot affect fiber Bragg-based sensors. Use in applications in potentially explosive atmospheres is also possible without restrictions.

In addition, piezoelectric sensors are very insensitive against high current in their environment.

\section{Linearity}

Electrical strain gauges attain high linearity so that normally no compensation is required. Nonlinearity errors of a few ppm relative to full scale are not a problem today.

$\begin{array}{lll}\text { Sample } & \begin{array}{l}\text { Gauge } \\ \text { Factor } \\ \text { Positive } \\ \text { Strain }\end{array} & \begin{array}{l}\text { Gauge } \\ \text { Factor } \\ \text { Negative } \\ \text { Strain }\end{array} \\ 1 & 0.808 & 0.79 \\ 2 & 0.804 & 0.785 \\ 3 & 0.801 & 0.784 \\ 4 & 0.804 & 0.781 \\ 5 & 0.808 & 0.794 \\ 7 & 0.806 & 0.793 \\ 8 & 0.803 & 0.79 \\ \text { Mean } & & \\ \text { Value } & \mathbf{0 . 8 0 5} & \mathbf{0 . 7 8 8}[\mathrm{HS} \text { ]] }\end{array}$

Table 1: Gauge factors of optical strain gauges of fiber Bragg sensors with tensile and compressive load

Piezoelectric sensors achieve a nonlinearity in the range of $0.5 \%$.

Optical strain gauges show a different gauge factor for tension and compression. 


\section{Conclusions}

This paper shows that different sensor types are suitable for different applications. Piezoelectric sensors have advantages for dynamic measurement tasks especially for low forces.

Strain gauge based sensors feature impressive accuracy also in harsh environments and the sensors are long-term stable in terms of zero point and sensitivity. The Wheatstone bridge allows to compensate many influences.

Optical sensors can be used when drift-free measurement is required and a strain gauge based system cannot be used, e.g., in environments with extreme electromagnetic fields or harsh chemical conditions.

\section{Refrences}

[1] Keil, S. Beanspruchungsanalyse mit -

Dehnungsmessstreifen, Cuneus-Verlag, 1995, ISBN 3-98041880-4

[2] Kreuzer, M. Strain Measurement with Fibre Bragg Grating Sensors, HBM, Darmstadt, S2338-1.0e

[3] Trutzel, M., Dejhnungsermittlung mit faseroptischen Bragg-Gitter-Sensoren, Berlin 2001, D83, Dissertation

[4] VDI/VDE 2635, Dehnungsmessstreifen mit metallischen Messgitter - Kenngrößen und Prüfbedingungen

[5] Fibre Bragg Sensors Compared With Electrical Strain Gauges for Use In Force Measurement Prospects and Potentials; XIX IMEKO World Congress Lisbon, 2009. Presentation. Thomas Kleckers 